\title{
Clonal Analysis of Micronodules in Virus C-Induced Liver Cirrhosis Using Laser Capture Microdissection (LCM) and HUMARA Assay
}

\author{
Valérie Paradis, Delphine Dargere, Franck Bonvoust, Laura Rubbia-Brandt, \\ Nathalie Bâ, Paulette Bioulac-Sage, and Pierre Bedossa
}

Service d'Anatomie Pathologique (VP, NB, PB), Hôpital de Bicêtre, Le Kremlin-Bicêtre, and UPRES A 8067 Faculté de Pharmacie (VP, DD, FB, PB), Paris, France; and Institut de Pathologie (LR-B), Geneva, Switzerland; and Service d'Anatomie Pathologique (PB-S), CHU Pellegrin, Bordeaux, France

\begin{abstract}
SUMMARY: Most hepatocellular carcinomas (HCC) arise from malignant transformation of regenerative cirrhotic nodules. Because $\mathrm{HCC}$ has a very poor prognosis, detection of these premalignant lesions may improve the management of patients with cirrhosis. In this regard, clonal analysis of liver micronodules should be of particular interest in order to differentiate polyclonal regenerative micronodules from monoclonal neoplastic potentially malignant micronodules. To address this issue, 112 micronodules from 15 cases of explanted liver cirrhosis were carefully microdissected from paraffin-embedded tissue using a laser capture microscopy system. Clonal analysis was performed by analyzing X-chromosome inactivation, as indicated by the methylation status of the human androgen receptor gene (HUMARA). For each microdissected micronodule, a large set of pathological features was evaluated and correlated with their clonal status. Clonal analysis showed that 57 micronodules (51\%) were monoclonal and 55 (49\%) were polyclonal. Prevalence of monoclonal nodules ranged from $25 \%$ to $71 \%$ according to cases. In all cases, mono- and polyclonal nodules were randomly distributed in the cirrhotic liver. Although the clonal status was not significantly affected by the presence or absence of macronodules in the adjacent liver, size of monoclonal micronodules was significantly larger than size of polyclonal micronodules (mean size of the monoclonal nodules: $3+0.1 \mathrm{~mm}$ vs mean size of the polyclonal nodules: $2.5 \pm 0.1 \mathrm{~mm}, p=0.007$ ). Among the elementary pathological features evaluated, only the presence of iron overload was correlated with a monoclonal status $(p=0.04)$. In conclusion, clonal analysis of liver cirrhosis shows that $51 \%$ of micronodules are monoclonal lesions, supporting the notion that liver cirrhosis is a multineoplastic lesion. Because monoclonality is a marker of neoplasia, cirrhosis with accumulation of monoclonal nodules may be carefully followed, and monoclonal nodules should be screened for additional markers to assess their biological behavior. (Lab Invest 2000, 80:1553-1559).
\end{abstract}

I $\mathrm{t}$ is generally admitted that tumors arise from clonal expansion of a single cell (Fialkow, 1976). Therefore, clonal analysis of various lesions, the neoplastic nature of which is unclear, is a powerful tool for assessing their biological behavior. Such an approach has been used to show that various lesions, including colon adenomas, breast atypical ductal hyperplasia, and liver adenomas, resulted from clonal cell proliferation consistent with a neoplastic disease (Noguchi et al, 1993; Paradis et al, 1997; Vogelstein et al, 1985). By contrast, the same technique has shown that, in the liver, focal nodular hyperplasia is a polyclonal regenerative lesion (Paradis et al, 1997).

To analyze clonality, the study of the $X$-chromosome inactivation pattern is a technique of choice, and use of the human androgen receptor gene

Received July 20, 2000.

This work was supported by grants from the Association pour la Recherche contre le Cancer and the Comité des Hauts-De-Seine de la Ligue contre le Cancer.

Address reprint requests to: Dr. Valérie Paradis, Service d'Anatomie Pathologique, Hôpital de Bicêtre, 78, rue du Gal Leclerc, 94275 Le Kremlin-Bicêtre, Paris, France. Fax: 331452132 81; E-mail: vparadis@teaser.fr
(HUMARA) as a marker of clonality is of major interest because of its high polymorphism (Busque et al, 1994). This technique is based on random inactivation by methylation of one of the two $\mathrm{X}$ chromosomes occurring in all female somatic cells during embryogenesis (Vogelstein et al, 1985, 1987). Since this inactivated condition is maintained through subsequent cell divisions regardless of acquired genetic changes, a monoclonal cell population will show a unique methylation pattern in all cells, whereas a polyclonal cell population will display different methylation patterns from one cell to another. We and others have shown that this technique can be validly performed on fixed paraffin-embedded tissue allowing retrospective studies (Mashal et al, 1993; Paradis et al, 1997, 1998; Quade et al, 1997). Until now, the limiting step of the analysis was related to the adequate and accurate tissue sampling of small lesions. Recent advances in micromanipulation technology, such as a laser capture microscopy (LCM) system, allow an accurate selection and a better capture of cell clusters of interest from tissue sections (Bernsen et al, 1998; Bonner et al, 1997; Emmert-Buck et al, 1996; Sirivatanauksorn et el, 1999). 
Hepatocellular carcinoma (HCC) is one of the leading causes of death in patients with chronic liver diseases and cirrhosis. Most cases arise from malignant transformation of regenerative cirrhotic nodules, and since HCC has a very poor prognosis, detection of precancerous monoclonal lesions may help in the management of patients with chronic liver diseases and cirrhosis. It has been shown that, among cirrhotic nodules, macronodules defined as being at least twice as large as nodules of the adjacent parenchyma may be considered as potential premalignant lesions (Ferrell et al, 1992; Furuya et al, 1988; Kondo et al, 1990; Okuda, 1992; Theise et al, 1992). Indeed, in a previous study analyzing the clonal pattern of a set of macronodules isolated from explanted liver cirrhosis, we found that $54 \%$ of them were monoclonal in origin, suggestive of neoplasia. Interestingly, we did not find any correlations with the usual histological classification, indicating the poor prognostic value of pathological classifications and highlighting the interest of further molecular approaches (Paradis et al, 1998).

Although some HCCs may develop from large-sized nodules, there is some evidence that they could also directly arise from smaller cirrhotic micronodules. Because their small size does not permit the detection of these minute, potentially premalignant lesions, it is important to set up surrogate markers that are able to predict the further development of HCC directly in this context. As for macronodules, some cirrhotic micronodules might display a monoclonal pattern and then behave as potentially malignant lesions. To explore this hypothesis, we investigated the clonal pattern of micronodules in explanted liver cirrhosis and compared these results with their histological features. This molecular approach should provide useful additional criteria for assessing the prognosis of cirrhosis.

\section{Results}

\section{Clinicopathological Features}

A total of 112 micronodules were microdissected from 15 cases of explanted liver cirrhosis, with 5 to 21 micronodules per case. All cases of cirrhosis were related to hepatitis $C$ virus infection. The main clinicopathological data are summarized in Table 1. The mean size of the 112 micronodules was $2.8 \pm 0.9 \mathrm{~mm}$ (ranges, 1-5 mm). Among them, 80 were obtained from 12 cases of explanted liver cirrhosis with macronodules and 32 from 3 cases of liver cirrhosis without macronodules. Eleven macronodules were also studied (mean size, $11.5 \mathrm{~mm}$; ranges, 8-15 mm). According to their histology, macronodules were classified as follows: four regenerative macronodules, four low-grade dysplastic macronodules, two high-grade dysplastic macronodules, and one malignant macronodule.

Mean size of micronodules was not significantly different, whether a macronodule was present or not in the adjacent cirrhotic liver $(2.9 \pm 0.9 \mathrm{~mm}$ vs $2.5 \pm$ $0.8 \mathrm{~mm}, p=\mathrm{NS}$ ). The prevalence of each histological elementary feature is reported in Table 2. No statistical difference was observed in the distribution of each pathological feature whether a macronodule was present or not in the surrounding tissue.

\section{Clonal Analysis}

DNA from the micronodules and adjacent liver was successfully extracted in all cases. Among the 112 micronodules studied, 57 (51\%) had a final ratio equal to or greater than 1.5, indicating a monoclonal pattern according to the titration curve. Representative profiles of monoclonal and polyclonal micronodules are shown in Figure 1. In all cases of cirrhosis, a mixture of mono- and polyclonal micronodular lesions was observed, with a prevalence of monoclonal nodules ranging from $25 \%$ to $71 \%$. In all cases, topographic distribution showed a random distribution of monoand polyclonal nodules in cirrhotic livers (Fig. 2).

Clonal analysis was also performed with the 11 macronodules dissected. Five of them (45\%) displayed a monoclonal pattern consistent with neoplasia. According to the histological classification, the

Table 1. Clinico-Pathological Data from 15 Cases of Explanted Liver Cirrhosis

\begin{tabular}{ccccc}
\hline Case & $\begin{array}{c}\text { Age } \\
(\mathrm{yr})\end{array}$ & $\begin{array}{c}\text { Presence of } \\
\text { macronodules }\end{array}$ & $\begin{array}{c}\text { Number of microdissected } \\
\text { micronodules }\end{array}$ & $\begin{array}{c}\text { Percentage of } \\
\text { monoclonal nodules }\end{array}$ \\
\hline 1 & 58 & No & 21 & 66 \\
2 & 48 & No & 5 & 60 \\
3 & 57 & No & 6 & 50 \\
4 & 52 & Yes & 8 & 38 \\
5 & 64 & Yes & 5 & 60 \\
6 & 66 & Yes & 7 & 71 \\
7 & 43 & Yes & 12 & 58 \\
8 & 35 & Yes & 7 & 57 \\
9 & 48 & Yes & 10 & 40 \\
10 & 55 & Yes & 4 & 25 \\
11 & 59 & Yes & 6 & 43 \\
12 & 54 & Yes & 5 & 20 \\
13 & 55 & Yes & 5 & 60 \\
14 & 63 & Yes & 5 & 33 \\
15 & 52 & Yes & 6 & \\
\hline
\end{tabular}


Table 2. Prevalence of Each Histological Elementary Feature in Cases of Explanted Liver Cirrhosis with Macronodules vs Cases of Liver Cirrhosis without Macronodules

\begin{tabular}{lrrrrr}
\hline & \multicolumn{2}{c}{$\begin{array}{c}\text { Cirrhosis with } \\
\text { macronodules }\end{array}$} & & \multicolumn{2}{c}{$\begin{array}{c}\text { Cirrhosis without } \\
\text { macronodules }\end{array}$} \\
\cline { 2 - 3 } \cline { 5 - 6 } Histological elementary feature & $\%$ & $(\mathrm{n}=80)$ & & $\%$ & $(\mathrm{n}=32)$ \\
\hline Fibrous septa & 79 & $(63)$ & & 50 & $(16)$ \\
Inflammatory infiltrate & 56 & $(45)$ & & 78 & $(25)$ \\
Increase in cell plate thickness & 89 & $(71)$ & & 75 & $(24)$ \\
Pseudo-glandular formations & 26 & $(21)$ & & 50 & $(16)$ \\
Isolated arteries & 15 & $(12)$ & & 12.5 & $(4)$ \\
Small liver cell change & 0 & $(0)$ & & 0 & $(0)$ \\
Large liver cell change & 5 & $(4)$ & & 0 & $(0)$ \\
Anisokaryosis & 30 & $(24)$ & & 31 & $(10)$ \\
Anisocytosis & 63 & $(50)$ & & 31 & $(10)$ \\
Iron overload & 10 & $(8)$ & & 34 & $(11)$ \\
Steatosis & 38 & $(30)$ & & 16 & $(5)$ \\
Presence of oncocytes & 30 & $(24)$ & & 0 & $(0)$ \\
\hline
\end{tabular}

monoclonal macronodules consisted of two regenerative macronodules, one low-grade dysplastic macronodule, one high-grade dysplastic macronodule, and one malignant macronodule.

\section{Correlations of Clonal Status with Pathological Data}

A positive correlation was observed between size and monoclonal pattern. The mean size of monoclonal micronodules was significantly higher than that of polyclonal micronodules $(3 \pm 0.1 \mathrm{~mm}, 2.5 \pm 0.1 \mathrm{~mm}$, $p=0.007$ ). Detailed data are shown in Table 3. Among the pathological features analyzed, the presence of iron overload was positively correlated with the monoclonal pattern. Fourteen out of 57 monoclonal nodules (25\%) had iron overload versus 5 out of 55 polyclonal nodules $(9 \%)(p=0.04)$. Inflammation in the nodule was inversely correlated with monoclonality. Forty out of 55 polyclonal nodules $(73 \%)$ had inflammation versus 30 out of 57 monoclonal nodules (53\%) $p=$ 0.03). The distribution of other histopathological features did not vary significantly with clonal status. Also, clonal status did not vary significantly according to the presence or absence of macronodules in the adjacent liver $(46 \%$ of micronodules were monoclonal in the group of cirrhosis cases without macronodules vs $62.5 \%$ in the group of cirrhosis cases with macronodules in the cirrhotic liver, $p=N S$ ).

\section{Discussion}

Liver cirrhosis is characterized by annular fibrosis delineating liver cell nodules defined according to their size as micro- and macronodules. The malignant potential of these nodular lesions has remained unclear for a long time. However, recent development of molecular tools allows a better characterization of their behavior, including the ability to differentiate polyclonal hyperplastic regenerative nodules from monoclonal neoplastic tumor nodules. In a previous study, we were able to demonstrate that approximately $50 \%$ of large macronodules obtained from samples of explanted liver cirrhosis were monoclonal according to the HUMARA polymorphism, consistent with their neoplastic nature (Paradis et al, 1998). In the present work, we applied the same approach to the study of small liver micronodules. Interestingly, we observed very similar results, ie, $51 \%$ of the micronodules included in the present study were monoclonal. Although monoclonality is a marker of neoplasia, it does not completely reflect the behavior of the lesion. Whether or not HCC arises from monoclonal nodules, acquisition of further properties, such as angiogenesis and invasion, is required for a monoclonal nodule to become malignant. In light of the relatively low percentage of cirrhosis cases that develop HCC $(5 \%$ to $20 \%$ ), such additional factors may appear in a limited number of monoclonal nodules. However, because the ratio of monoclonal nodules is highly variable $(20 \%$ to $70 \%$ ) among cases of cirrhosis, it can be reasonably suggested that cirrhoses having a high percentage of monoclonal nodules are at higher risk of developing a cancer. Nevertheless, such a hypothesis must be confirmed by further studies. In addition, the monoclonality ratio was not significantly different, regardless of the presence or absence of macronodules in the adjacent liver. Our results indicate that clonal expansion may occur early in the cirrhotic liver, even before the development of large macronodules. This is in agreement with previous observations suggesting that HCC could directly occur, in the context of hepatitis $C$ virus infection, on the background of liver cirrhosis without the previous development of macronodules.

According to previous studies, the prevalence of monoclonal nodules in cirrhosis varied from $0.5 \%$ to 59\% (Aihara et al, 1996; Ochiai et al, 2000). Such discrepancies may depend at least partly on technical drawbacks. To assess the clonality of lesions, one major prerequisite is the accuracy of the sampling, because the main limitation of this technique lies in the potential contamination of cells of interest (hepatocytes) by other cells, including inflammatory or stromal 

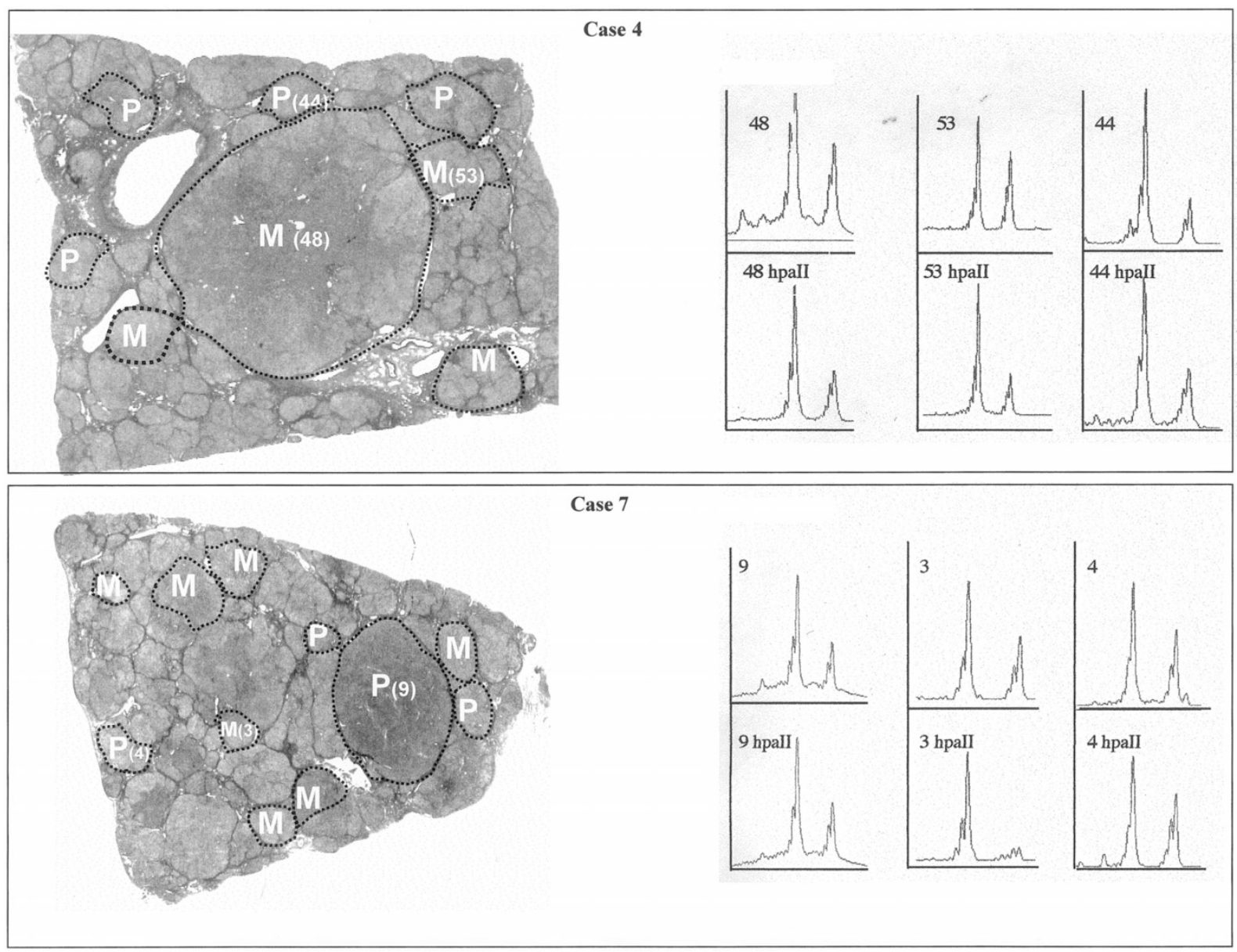

\section{Figure 1.}

Clonal analysis of microdissected liver nodules in 2 cases of cirrhosis. Upper panel, Cirrhosis Case 4: methylation patterns without digestion (top) or after Hpall digestion (bottom) of the macronodule (48) and two micronodules (53 and 44) are shown. A significant decrease of one of the alleles is observed after Hpall digestion in nodules 48 and 53, suggestive of monoclonality (M). Nodule 44 exhibits the same methylation pattern without digestion or after Hpall digestion, suggestive of polyclonality $(\mathrm{P})$. Lower panel, Cirrhosis Case 7: macronodule $(9)$ and nodule 4 are polyclonal $(\mathrm{P})$ since both of them display the same methylation pattern without digestion or after Hpall digestion. Only one peak is observed after Hpall digestion in nodule 3, suggestive of monoclonality (M).

cells. To study the clonality of very small lesions such as liver micronodules, the use of tissue microdissection is mandatory. For this purpose we used a laser capture microscopy system, though contamination of hepatocytes with sinusoidal cells or inflammatory cells cannot be completely avoided. This contamination is shown, in the present study, by the significant association of inflammation as a histological criterion with the polyclonal status of the micronodules. In any case, the capture of groups of hepatocytes delineated by the fibrous septa was easy to perform reproducibly. Furthermore, the use of paraffin-embedded tissue facilitates the procedure because liver morphology is well-conserved allowing an accurate delimitation of each nodule of interest. In our hands, liver nodule microdissection provided high-quality DNA suitable for clonal analysis. However, the need for a certain amount of DNA for molecular analysis limits the study to nodules of a minimal size of approximately $1 \mathrm{~mm}$.

When analyzing correlations between clonal pattern and various parameters, we observed that monoclonal micronodules were significantly larger than polyclonal micronodules. Based on this observation, we can postulate that enlargement of micronodules is related to clonal expansion of a group of monoclonal liver cells rather than to the multiplication of regenerative polyclonal cells. In a previous study showing that $43 \%$ of regenerative nodules in hepatitis $C$ virus-induced liver cirrhosis were monoclonal, it was suggested that monoclonal nodules are derived from separation by fibrous septa of larger monoclonal cell clusters (Aihara et al, 1994). Such a hypothesis would result in the juxtaposition of monoclonal nodules. By showing random distribution of poly- and monoclonal nodules within the cirrhotic liver, our study does not support such a hypothesis.

It has been previously shown that liver tissue from chimeric animals is composed of the apposition of cell clusters (patches) of similar genetic background (Howell et al, 1985; Weinberg et al, 1985). In humans, a recent study also suggested the presence of patches in liver tissue. However, the estimated mean size of these monoclonal patches $\left(1 \mathrm{~mm}^{2}\right)$ was smaller than the mean size of a cirrhotic micronodule included in our study (Ochiai et al, 2000). This data strongly supports the hypothesis that monoclonal micronod- 


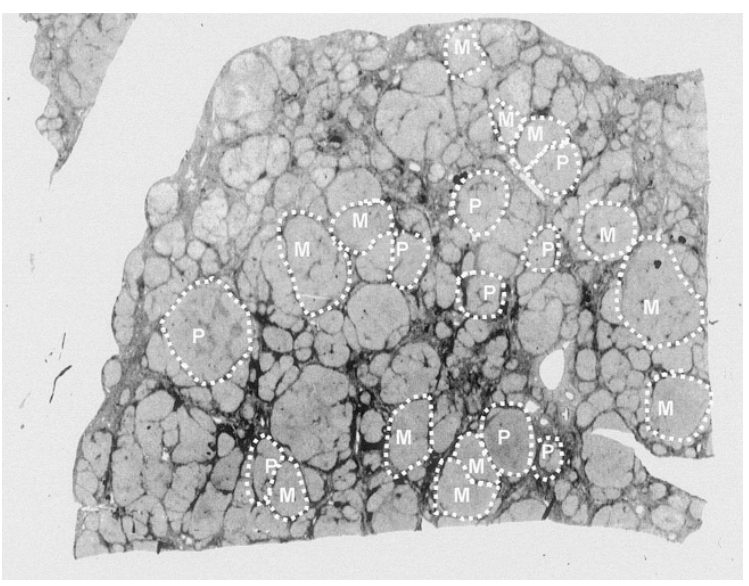

Figure 2.

Distribution of mono- and polyclonal micronodules microdissected from a liver cirrhosis (Case 3). Clonal analysis showed a random distribution of mono- (M) and polyclonal $(\mathrm{P})$ micronodules in a case of cirrhosis without macronodule.

ules do not proceed from the isolation by fibrous tissue of physiological monoclonal patches of the normal liver and suggests that monoclonal micronodules derive from clonal expansion of cells or groups of neighboring cells

$\mathrm{HCC}$, one of the most common malignancies, is associated with a poor prognosis. Since most HCCs develop in the course of closely followed-up cirrhotic patients, detection of early neoplastic lesions could significantly improve the outcome in patients with liver cirrhosis. However, the follow-up of patients with liver cirrhosis remains difficult and is mainly based on imaging screening. Because a certain number of the monoclonal micronodular lesions cannot be detected by imaging approaches, it is crucial to develop additional tools to identify potential premalignant lesions. Among these additional tools, histological analysis could provide reliable information. To identify monoclonal lesions on pathological grounds, a large set of morphological features, including cytological and architectural criteria, were screened in this series and correlated with the clonal pattern of each micronodule. Our data showed that the presence of iron overload was the only pathological feature that correlated with the monoclonal pattern. Such a result is supported by several reports. Indeed, recent data showed that dietary iron overload in animals results in an increased number of preneoplastic foci (Stal et al, 1999). Furthermore, in the literature, a carcinogenic effect of iron overload has already been suggested (Deugnier et al, 1993; Kew, 1990).
In conclusion, our study shows that $20 \%$ to $70 \%$ of small-sized liver cell nodules in a cirrhotic liver are monoclonal according to the HUMARA assay. Whether this test can be used as a potential marker of the future development of a carcinoma will require further studies.

\section{Materials and Methods}

A total of 112 micronodules, defined as liver cell nodules less than $5 \mathrm{~mm}$ in diameter, were microdissected from explanted liver cirrhosis in 15 women. From these, 80 micronodules were obtained from 12 cases of liver cirrhosis also having macronodules, but without HCC. The 32 other micronodules were from 3 patients with uniform micronodular cirrhosis without any macronodules or HCC. All explanted livers were serially sectioned into thin slices, formalin-fixed, paraffin-embedded, and routinely processed. Histological examination was assessed on hematoxylin and eosin (H\&E)-stained sections by two pathologists. For each micronodule studied, 13 elementary features (fibrous septa, inflammatory infiltrates, increase in cell plate thickness, pseudo-glandular formations, isolated arteries, small liver cell change, large liver cell change, anisokaryosis, anisocytosis, iron overload, steatosis, presence of oncocytes) were systematically analyzed and semiquantitatively assessed. Macronodules, when present, were grouped into four categories according to the international consensus: regenerative macronodule (benign), low grade dysplastic macronodule, high grade dysplastic macronodule, and malignant macronodule (HCC) (International Working Party, 1995).

\section{Laser Capture Microdissection (LCM)}

Paraffin-embedded blocks of cirrhotic tissue were serially cut. For each case, the first $5-\mu \mathrm{m}$ section was stained with H\&E for histological analysis and digitalized. For LCM, a following $16-\mu \mathrm{m}$ serial section was cut and mounted on an uncharged glass slide. The slide was deparaffinized in xylene for 30 minutes and then air-dried. Each micronodule was microdissected by LCM with a PixCell instrument (Arcturus Engineering, Mountain View, California) after being identified in the adjacent H\&E-stained section. Briefly, the LCM system uses an ethylene vinyl acetate transfer film coated onto a 6-mm-diameter rigid flat cap. Under the microscope, the nodule of interest can be viewed through the film, and activation of the pulsed laser beam results in melting the film directly above the

Table 3. Mean Size of Micronodules According to Clonal Analysis in Cases of Explanted Liver Cirrhosis with Macronodules vs Cases of Liver Cirrhosis without Macronodules

\begin{tabular}{llll}
\hline & $\begin{array}{c}\text { Polyclonal } \\
\text { micronodules }\end{array}$ & $\begin{array}{c}\text { Monoclonal } \\
\text { micronodules }\end{array}$ & $p$ \\
\hline Cirrhosis with macronodules & $2.6 \pm 0.1 \mathrm{~mm}$ & $3.1 \pm 0.1 \mathrm{~mm}$ & 0.03 \\
Cirrhosis without macronodules & $2.1 \pm 0.1 \mathrm{~mm}$ & $2.8 \pm 0.2 \mathrm{~mm}$ & 0.01 \\
All cirrhosis & $2.5 \pm 0.1 \mathrm{~mm}$ & $3 \pm 0.1 \mathrm{~mm}$ & 0.007 \\
\hline
\end{tabular}


targeted cells. For each nodule, the parameters used included a laser diameter of $30 \mu \mathrm{m}$, laser power of 50 $\mathrm{mW}$, and a pulse length of $10 \mathrm{~ms}$. After visual control of the completeness of dissection, the cap with the captured micronodular tissue was placed onto a 0.5 $\mathrm{ml}$ microfuge tube containing $200 \mu$ l of proteinase $\mathrm{K}$ buffer. The tube was inverted and incubated overnight at $37^{\circ} \mathrm{C}$. After inactivation at $95^{\circ} \mathrm{C}$ for 10 minutes, the sample was ready for DNA extraction (phenol/chloroform extraction).

\section{Assessment of Clonality}

Clonality at the HUMARA locus was assessed by PCR amplification as previously described (Vogelstein et al, 1985). Briefly, the HUMARA gene includes a polymorphic [(CAG)n] repeat located $3^{\prime}$ of the methylationsensitive Hpall restriction-enzyme sites. The PCR assay uses primers whose product spans both the Hpall sites and the [(CAG)n] polymorphism. Variations in the lengths of the $[(C A G) n]$ repeats on the paternal and maternal $X$ chromosomes will yield HUMARA alleles of different lengths. Methylation of the Hpall sites will distinguish the active (nonmethylated) from the inactive (methylated) $X$ chromosome. Only the inactive methylated $X$ chromosome will not be digested by Hpall and thus amplified by PCR.

Samples of DNA solution were digested overnight at $37^{\circ} \mathrm{C}$ in the presence or absence of Hpall (10 units/ tube; Boehringer Mannheim $\mathrm{GmbH}$, Mannheim, Germany) and then heated at $95^{\circ} \mathrm{C}$ for 10 minutes to inactivate Hpall. Hpall-digested or nondigested DNA samples ( $2 \mu \mathrm{l}$ each) were used for amplification of HUMARA by adding to $18 \mu \mathrm{l}$ of PCR reaction mixture containing $2 \mu \mathrm{l}$ of PCR buffer $10 \times, 1 \mu \mathrm{l}$ of $\mathrm{MgCl}_{2} 25$ $\mathrm{mm}, 2 \mu \mathrm{l}$ each of dNTP $(200 \mu \mathrm{M}), 1 \mu \mathrm{l}$ each of primer (10 pmol), $0.3 \mu \mathrm{l}$ of AmpliTaq GoldTM DNA polymerase (Perkin-Elmer Cetus, Norwalk, Connecticut), and $10.7 \mu \mathrm{l}$ of de-ionized $\mathrm{H}_{2} \mathrm{O}$. The sequences of the primers used for amplification of HUMARA DNA were 5'GCTGTGAAGGTTGCTGTTCCTCAT3' and 5'TCCAGAATCTGTTCCAGAGCGTGC 3' (Noguchi et al, 1993). Primer 1 was labeled at the 5 ' end with fluoroscein. Initial denaturation was performed for 10 minutes at $94^{\circ} \mathrm{C}$, followed by 30 cycles of 30 seconds at $94^{\circ} \mathrm{C}, 30$ seconds at $60^{\circ} \mathrm{C}$, and 1 minute at $72^{\circ} \mathrm{C}$. In the final cycle, extension at $72^{\circ} \mathrm{C}$ was prolonged for 7 minutes. All PCR samples were run in duplicate.

After amplification, $3 \mu$ l of the PCR products were mixed with $2.6 \mu \mathrm{l}$ of loading buffer containing internal size standards (Genescan 500 Rox; Applied Biosystems, Foster City, California) and electrophoresed through a $6 \%$ denaturing gel run on a 373A DNA sequencer (Applied Biosystems). The relative amount of PCR products (area under the curve) was calculated for each allele using Genescan 672 Software (Applied Biosystems). A corrected ratio (CR) was first assessed by dividing the ratio (allele 1/allele 2) of the digested sample obtained after digesting DNA with Hpall by the ratio (allele 1/allele 2 ) of the nondigested sample. The use of CR corrects for the preferential amplification of one allele that might occur if the alleles differ markedly in length. A final clonality ratio for each tumor was determined by dividing the $\mathrm{CR}$ of the micronodule DNA by the CR of the adjacent liver DNA. This final clonal ratio corrects for the potential skewed lyonization. To estimate the percentage of clonal cells in the lesion according to the value of the final ratio, a titration curve was performed as previously described (Paradis et al, 1997). A lesion was considered as monoclonal when it consisted of at least $25 \%$ of monoclonal cells in a polyclonal background. According to the titration curve we performed, this percentage corresponded to a final ratio of 1:5.

\section{Statistics}

Distributions between groups were compared using the $\chi^{2}$ test or Fisher exact test for qualitative data and the Student $t$ test for quantitative data. The $p$ value of 0.05 was chosen as the significant level.

\section{References}

Aihara T, Noguchi S, Sasaki Y, Nakano H, and Imaoka S (1994). Clonal analysis of regenerative nodules in hepatitis $C$ virus-induced liver cirrhosis. Gastroenterology 107:18051811.

Aihara T, Noguchi S, Sasaki Y, Nakano H, Monden M, and Imaoka S (1996). Clonal analysis of precancerous lesion of hepatocellular carcinoma. Gastroenterology 111:455-461.

Bernsen MR, Dijkman HB, de Vries E, Figdor CG, Ruiter DJ, Adema GJ, and van Muijen GN (1998). Identification of multiple mRNA and DNA sequences from small tissue samples isolated by laser-assisted microdissection. Lab Invest 78:1267-1273.

Bonner RF, Emmert-Buck MR, Cole K, Pohida T, Chuaqui R, Goldstein S, and Liotta LA (1997). Laser capture microdissection. Molecular analysis of tissue. Science 278:14811483.

Busque L, Zhu J, Dehart D, Griffith B, Willman C, Carroll R, Black PM, and Gilliland DG (1994). An expression based clonality assay at the human receptor locus (HUMARA) on chromosome X. Nucleic Acids Res 22:697-698.

Deugnier Y, Charalambous P, Le Quilleuc D, Turlin B, Searle J, Brissot P, Powell LW, and Halliday JW (1993). Preneoplastic significance of hepatic iron-free foci in genetic hemochromatosis: A study of 185 patients. Hepatology 18: 1363-1369.

Emmert-Buck MR, Bonner RF, Smith PD, Chuaqui RF, Zhuang Z, Goldstein SR, Weiss RA, and Liotta LA (1996). Laser capture microdissection. Science 274:998-1001.

Ferrell L, Wright T, Lake J, Roberts J, and Ascher N (1992). Incidence and diagnostic features of macroregenerative nodules vs small hepatocellular carcinoma in cirrhotic livers. Hepatology 16:1372-1381.

Fialkow PJ (1976). Clonal origin of human tumors. Biochem Biophys Acta 458:283-321.

Furuya K, Nakamura M, Yamamoto Y, Togei K, and Otsuka H (1988). Macroregenerative nodule of the liver: A clinicopathologic study of 345 autopsy cases of chronic liver disease. Cancer 61:99-105. 
Howell S, Wareham KA, and Williams ED (1985). Clonal origin of mouse liver cell tumors. Am J Pathol 121:426-432.

International Working Party (1995). Terminology of nodular hepatocellular lesions. Hepatology 22:983-993.

Kew M (1990). Pathogenesis of hepatocellular carcinoma in hereditary hemochromatosis: Occurrence in noncirrhotic liver. Hepatology 11:1086-1087.

Kondo F, Ebara M, Sugiura N, Wada K, Kita K, Hirooka N, Nagoto Y, Kondo Y, Ohto M, and Okuda K (1990). Histological features and clinical course of large regenerative nodules: Evaluation of their precancerous potentiality. Hepatology 12:592-598.

Mashal RD, Lester SC, and Sklar J (1993). Clonal analysis by study of $X$ chromosome inactivation in formalin-fixed paraffin-embedded tissue. Cancer Res 53:4676-4679.

Noguchi S, Motomura K, Inaji H, Imaoka S, and Koyama H (1993). Clonal analysis of predominantly intraductal carcinoma and precancerous lesions of the breast by means of polymerase chain reaction. Cancer Res 53:4071-4074.

Ochiai T, Urata Y, Yamano T, Yamagishi H, and Ashihara T (2000). Clonal expansion in evolution of chronic hepatitis to hepatocellular carcinoma as seen at an X-chromosome locus. Hepatology 31:615-621.

Okuda K (1992). Hepatocellular carcinoma: Recent progress. Hepatology 15:948-963.

Paradis V, Laurent A, Flejou JF, Vidaud M, and Bedossa P (1997). Evidence for the polyclonal nature of focal nodular hyperplasia of the liver by the study of X-chromosome inactivation. Hepatology 26:891-895.

Paradis V, Laurendeau I, Vidaud M, and Bedossa P (1998). Clonal analysis of macronodules in cirrhosis. Hepatology 28:953-958.
Quade BJ, McLachlin CM, Soto-Wright V, Zuckerman J, Mutter GL, and Morton CC (1997). Disseminated peritoneal leiomyomatosis: Clonality analysis by $\mathrm{X}$ chromosome inactivation and cytogenetics of a clinically benign smooth muscle proliferation. Am J Pathol 150:2153-2166.

Sirivatanauksorn Y, Sirivatanauksorn V, Bhattacharya S, Davidson $B R$, Dhillon AP, Kakkar AK, Williamson RCN, and Lemoine NR (1999). Evolution of genetic abnormalities in hepatocellular carcinomas demonstrated by DNA fingerprinting. J Pathol 189:344-350.

Stal P, Wang G-S, and Eriksson LC (1999). Effects of dietary iron overload on progression in chemical hepatocarcinogenesis. Liver 19:326-334.

Theise ND, Schwartz M, Miller C, and Thung SN (1992). Macroregenerative nodules and hepatocellular carcinoma in forty-four sequential adult liver explants with cirrhosis. Hepatology 16:949-955.

Vogelstein B, Fearon ER, Hamilton SR, and Feinberg AP (1985). Use of restriction fragment length polymorphisms to determine the clonal origin of human tumors. Science 227: 642-645.

Vogelstein B, Fearon ER, Hamilton SR, Preisinger AC, Willard HF, Michelson AF, Riggs AD, and Orkin SH (1987). Clonal analysis using recombinant DNA probes from the X-chromosome. Cancer Res 47:4806-4813.

Weinberg WC, Howard JC, and lannaccone PM (1985). Histological demonstration of mosaicism in a series of chimeric rats produced between congenic strains. Science 227:524-527. 